\title{
Fundamentos filosóficos y sociológicos de la inclusión educativa y la atención a la diversidad
}

\section{Philosophical and sociological foundations of educational inclusion and attention to diversity}

Martha María Fernández Rodríguez.

Instituto Superior Tecnológico Espíritu Santo, Ecuador

Autor para correspondencia: fmarthamaria@yahoo.es

Fecha de recepción: 30 de Agosto de 2016 - Fecha de aceptación: 25 de Octubre de 2016

\section{Resumen}

Se presentaron los Fundamentos filosóficos y sociológicos de la inclusión educativa y la atención a la diversidad. El objetivo de este trabajo fue dotar en la práctica educacional a los involucrados en la inclusión educativa y la atención a la diversidad, de manera que partan de los sustentos teóricos que le permitan conducir con mayor preparación dicho proceso. El contenido del artículo formó parte de una capacitación en Inclusión y atención a la diversidad que se efectúo a los profesores del Colegio Femenino Espíritu Santo de Guayaquil. Se emplearon métodos teóricos como el análisis y la síntesis, de lo abstracto a lo concreto y el histórico lógico. Se logró como resultado una mayor preparación en los capacitados al quedar preparados en los fundamentos filosóficos y sociológicos que conducen el proceso de inclusión y atención a la diversidad.

Palabras claves: filosóficos; sociológicos; legales; diversidad; inclusión

\begin{abstract}
:
The philosophical and sociological foundations of educational inclusion and attention to diversity were presented. The aim of this work was to provide educational practice to those involved in educational inclusion and attention to diversity, in this departing from the theoretical underpinnings which allow get a better preparation in this process. This article was part of a training for inclusion and attention to diversity given to the teachers at Colegio Femenino Espíritu Santo in Guayaquil. Theoretical methods such as analysis and synthesis, from the abstract to the concrete and induction and deduction were used. The achieved result for the trainees was a greater preparation in the philosophical and sociological fundamentals leading the process of inclusion and attention to diversity.
\end{abstract}

Key words: philosophical; sociological; legal; diversity; inclusion 


\section{Introducción}

La educación es un derecho que los Estados han de promover, respetar y garantizar a todas las personas a lo largo de su vida. Lograrlo supone que todos tengan acceso a una educación de calidad en igualdad de oportunidades y condiciones. Justamente son estos los aspectos que definen a la educación inclusiva, de manera que se disminuya la discriminación y exclusión; he ahí la importancia y actualidad del tema.

En los últimos tiempos en Ecuador, se acometen transformaciones encaminadas a lograr un proceso de inclusión coherente con las políticas internacionales y nacionales. Lo anteriormente expuesto, fundamenta la necesidad e importancia de que se preste especial atención a la capacitación teórica que exige dicho proceso para poderlo enfrentar en la práctica educacional.

De ahí, que se presenten los fundamentos filosóficos y sociológicos de la inclusión educativa y la atención a la diversidad. El propósito de este trabajo es, dotar de sustentos teóricos a los involucrados en la inclusión educativa y la atención a la diversidad, de manera que le permita en la práctica educacional conducir con mayor preparación dicho proceso. El contenido del artículo forma parte de una capacitación en Inclusión y Atención a la Diversidad que se efectuó en el Colegio Femenino Espíritu Santo de la ciudad de Guayaquil. Se emplean métodos teóricos como el análisis y la síntesis, de lo abstracto a lo concreto y el histórico-lógico.

\section{Metodología}

La investigación es de tipo explicativa y descriptiva ya que fundamenta desde el punto de vista filosófico y sociológico el proceso de inclusión y atención a la diversidad educativa en Ecuador como contenido esencial de la capacitación, que sirve de base epistemológica para la preparación de los involucrados en el proceso educativo, adscritos a las políticas educativas en el país.

Se emplearon métodos teóricos como el análisis y la síntesis, de lo abstracto a lo concreto y el histórico lógico en relación con los contenidos esenciales que caracterizan a la Filosofía de la Educación y las políticas educativas en Ecuador, que constituyen fundamentos filosóficos y sociológicos de la inclusión.

\section{Resultados}

El resultado esencial de este artículo se refleja en los fundamentos filosóficos y sociológicos de la inclusión y atención a la diversidad que constituye la esencia de la capacitación a los docentes.

Cuando hablamos de fundamentos filosóficos en el orden educativo, hay que referirse de manera obligada a la Filosofía de la Educación, que trata acerca del quehacer educativo; pero no de forma abstracta sino a partir de la propia práctica educativa. Por ende, la interrelación teoríapráctica como fundamento general ayuda a la Filosofía de la Educación a dar respuesta a los 
infinitos problemas educativos que le conciernen con posiciones teóricas sólidas sin abandonar la realidad educativa.

En todos los profesores ecuatorianos partícipes de una educación inclusiva surge la interrogante ¿qué hacer para educar íntegramente y lograr la inclusión y atención a la diversidad?; he ahí un primer aspecto que no se refiere solo a problemas didácticos y metodológicos sino:

A una racionalización superior que pondera una determinada concepción de la vida. Se encuentra en el marco de estas reflexiones además del cómo, el para qué, lo que precisa la necesidad del examen de las finalidades educativas en la que se enmarca la práctica pedagógica concreta en el ámbito axiológico, epistemológico, lógico y metodológico conceptual en general. (Martínez, M. y Rodríguez J, 2002, p.5)

Es por ello que "reflexionar sobre el sentido de la educación es función básica de la Filosofía de la Educación por tanto esa reflexión se vuelve infinita en tanto lo es así el desarrollo humano". (Martínez, M. y Rodríguez J, 2002, p.6). De ahí, que cuando se fundamenta filosóficamente la educación en Ecuador hay que hablar de la Educación Inclusiva que forma parte de su política educativa para todos los niveles.

La Filosofía de la Educación, es un fundamento básico de las ideas educativas y pedagógicas y constituye referencia obligada de todas los profesores, directivos y asesores, como única vía para apreciar los modelos educativos que rigen en las educaciones, interpretar convenientemente las fines de la educación en un tiempo y contexto determinado y para expresar, de manera coherente, las necesidades y posibilidades educativas de la sociedad y del individuo. A la luz de estas concepciones es que estamos siendo consecuente en Ecuador con un modelo educativo inclusivo que atienda a la diversidad.

Desde estos presupuestos se asume como Filosofía de la Educación:

Un saber general de los procesos y acciones educativas a partir de presupuestos antropológicos, epistemológicos, axiológicos con un enfoque crítico metodológico general o como una concepción general del proceso educativo que surge como resultado de la aplicación de un enfoque integrador, cosmovisivo y crítico axiológico de este proceso complejo y contradictorio en que se produce la formación del hombre mediante la asunción creadora de los valores de la cultura universal a partir de la identidad nacional y de la transformación práctica. (Martínez, M. y Rodríguez J, 2002, p.8)

Se asume esta definición en tanto dichos presupuestos garantizan un enfoque holístico del fenómeno educativo, de ahí que el objeto de la Filosofía de la Educación cuando se trata de Educación Inclusiva y atención a la diversidad hay que concretarlo en problemas relacionados que atañan a dicho proceso y que conllevan a la no exclusión. Desde esta perspectiva, una Filosofía de la Educación que sustente el proceso de inclusión y atención a la diversidad en Ecuador, debe comprender todos los problemas que conciernen a la formación del hombre, en su diversidad como proceso y resultado. 
En este sentido, según (Martínez, M. y Rodríguez J, 2002) se habla de tres dimensiones en el análisis: especulativa, la prescriptiva o normativa y la crítica o analítica:

La especulativa busca establecer teorías de la naturaleza del hombre, de la sociedad y del mundo (...), la prescriptiva o normativa específica los fines que la educación debe perseguir y medios para alcanzarlos (...) y la crítica o analítica examina la propia teoría y la racionalidad de los ideales educativos o sea corresponde a las bases conceptuales de la educación en lo que respecta a la enseñanza, el aprendizaje y el currículum" (Martínez, M. y Rodríguez J, 2002, p.5)

Siendo consecuente con estas dimensiones, Ecuador norma en su política educativa la inclusión y asienta las bases para su cumplimento en la práctica educativa. Para ello exige dentro de la Educación cada vez más ser consecuentes con la atención a la diversidad, situación que justifica la necesidad de que los profesores estén capacitados para llevar a cabo dicho proceso y que comprendan las políticas educativas para lograr una educación inclusiva de calidad.

Según (Martínez, M. y Rodríguez J, 2002), la Filosofía de la Educación se rige por principios como: carácter multinacional, sistémico, histórico y el humanismo.

El carácter multinacional se refiere a que en su análisis se requiere de la consideración de los diferentes aspectos filosóficos que atañen al fenómeno educativo en sí mismo como objeto de investigación y valoración.

El carácter sistémico como principio se apoya en que la consideración multifuncional se realiza coordinadamente de forma tal que cada uno de los elementos es parte del todo y la alteración o cambio de enfoque de alguno de ellos afecta al resto.

El carácter histórico se manifiesta en tanto en cuanto el momento específico de insertarse en el análisis social se condiciona por los sucesos, acontecimientos y enfoques de época que determinan la naturaleza del hombre a formar como fin básico de la educación.

El Humanismo se fundamenta en el hecho de que todo sistema educacional supone la formación de un ideal humano y la consideración de todos los factores que inciden en él como línea directriz del análisis y conformación de los basamentos teóricos y prácticos de la concepción que se asuma. (p.8)

Estos principios guían el accionar pedagógico en relación con la Educación Inclusiva y atención a la diversidad en Ecuador. Con respecto al carácter sistémico la Educación en Ecuador exige a todos los niveles ser consecuente con la inclusión, que no excluya razas, etnias, género, cultura, saberes y en la que se tenga en cuenta lo fines, principios, medios, lo instructivo, lo educativo y los valores como un todo en la que no se puede fragmentar ni ausentarse ninguno, sino el proceso educativo no logra ser inclusivo, de ahí, que se concreten en las políticas educativas en relación con el tema. El carácter histórico se refleja en tanto la época actual a nivel internacional exige una educación para todos, sin excluir a nadie como una manera de respeto a la individualidad y el principio humanismo en tanto centra su atención en la formación de un ideal humano en el que todos tengan las mismas oportunidades sin exclusión de ningún tipo. 
Desarrollar una educación inclusiva implica sensibilizar a los docentes de manera que se logre una empatía y valoración adecuada sobre la atención a la inclusión y la diversidad. Para lograr tal empeño la Vicepresidencia de La República y el Ministerio de Educación enfocan sus políticas y programas educativos inclusivos y se asume como Educación Inclusiva "la formulación y aplicación de una vasta gama de estrategias de aprendizaje que respondan precisamente a la diversidad de los educandos". (UNESCO, 2008, p.10)

Para alcanzar tal empeño hay que crear "entornos inclusivos, lo cual implica:

a. el respeto, la comprensión y la atención a la diversidad cultural, social e individual (respuesta de los sistemas educativos, escuelas y docentes a las expectativas y necesidades de los alumnos); b) el acceso en condiciones de igualdad a una educación de calidad; y c) la estrecha coordinación con otras políticas sociales. En todos estos puntos se deben tomar en cuenta las expectativas y las demandas de las partes interesadas y los actores sociales. (UNESCO, 2008, p.10)

La sociedad ecuatoriana acepta la inclusión, de ahí que le ofrece las mismas oportunidades sin distinción de sexo raza, etnia, capacidad física e intelectual, a todos sus miembros y crea las mismas oportunidades para todos, así queda plasmado por el Ministerio de Educación en su política de educación inclusiva, con sus principios y objetivos.

La educación inclusiva plantea:

- Acceso de niños, niñas y/o adolescentes con discapacidad a una educación formal de calidad.

- Apoyo al trabajo en equipo para lograr la inclusión.

- Una visión global de la atención educativa, es decir a nivel institucional, no como atención puntual para unos pocos estudiantes.

- Un desequilibrio entre las prácticas pedagógicas tradicionales y las prácticas innovadoras que permiten atender a la diversidad.

- Preparación de ambientes y generación de recursos para atender a la diversidad.

- Transformaciones profundas en el Proyecto Educativo Institucional que respondan a la diversidad.

- Trabajar con la comunidad en general, permitiendo una participación activa de todos sus miembros.

- Generar una concepción natural acerca de las personas con discapacidad.

- Desarrollar una comunidad basada en valores inclusivos como: la solidaridad, el respeto y la tolerancia. (Ministerio de Educación, 2011, p.28-29).

Por su parte los objetivos que guían la Educación Inclusiva en Ecuador son:

- Brindar respuestas apropiadas al amplio espectro de necesidades de aprendizaje tanto en entornos formales como no formales de la educación, representa una perspectiva que debe servir para analizar cómo transformar los sistemas educativos y otros entornos de aprendizaje, con el fin de responder a la diversidad de los estudiantes. El propósito de la educación inclusiva es permitir que los maestros y estudiantes se sientan cómodos ante la 
diversidad y la perciban no como un problema, sino como un desafío y una oportunidad para enriquecer el entorno de aprendizaje.

- Equivale a decir que todos los niños, niñas y/o adolescentes tienen las oportunidades para acceder a una educación de calidad, respetando las diferencias individuales para lograr ciudadanos incluidos en el contexto social. (Ministerio de Educación, 2011, p.30).

También de la Educación inclusiva en Ecuador, se rige por principios como:

- Igualdad: equivale a decir que todos los niños, niñas y/o adolescentes tienen las oportunidades para acceder a una educación de calidad, respetando las diferencias individuales para lograr ciudadanos incluidos en el contexto social.

- Comprensividad: es la necesidad de mantener por parte de la escuela un currículo básico y común en un período largo, sobre todo en la educación obligatoria, para atender la diversificación de los estudiantes en función de su origen económico, social y cultural.

- Globalización: es la visión mediante la cual se prepara al estudiante para enfrentarse con los problemas de la vida y desde distintas disciplinas curriculares. (Ministerio de Educación, 2011, p.30-31).

Con la capacitación se logra nutrir de fundamentos teóricos a los docentes de manera que queden preparados en los elementos básicos para poder en su práctica educativo lograr la inclusión y atención a la diversidad.

\section{Conclusiones}

La Filosofía es un fundamento esencial de las concepciones educativas y pedagógicas. Todos los involucrados en la labor educativa deben estar capacitados en los sustentos filosóficos y sociológicos que le permitan ejercer la profesión con una preparación sólida y poder cumplir con responsabilidad lo que la política educativa ecuatoriana exige.

Ecuador tiene dentro de sus políticas educativas la inclusión y la atención a la diversidad, así lo reflejan sus principios, objetivos y dimensiones, además del marco legal sustentado en la Constitución y los organismos rectores educativos.

\section{Bibliografía}

Martínez Llantada, M. y Jesús Rodríguez Coturruelo. (2002). Filosofía de la Educación. Recuperado de: es.slideshare.net/hansmejia/libro-filosofa-de-la-educacin

Ministerio de Educación. (2011). Módulo I: educación inclusiva y especial. Atención a la diversidad - Educación - Departamento de Educación. Recuperado de: educacion.gob.ec/recursos-para-docentes/

UNESCO. (2008). Conferencia Internacional de Educación "la educación inclusiva: el camino hacia el futuro" Recuperado de: www.ibe.unesco.org/.../user.../Confinted_483_Spanish.pdf 